\title{
Spray-wall interaction and mixing studies in a heated duct air flow
}

\author{
Palaniappan Venkatachalam*1, Kameswararao Anupindi ${ }^{2}$, Srikrishna Sahu ${ }^{3}$ \\ 1, 2, ${ }^{3}$ Department of Mechanical Engineering, IIT Madras, Chennai, India \\ ${ }^{*}$ Corresponding author email: me19s041@smail.iitm.ac.in
}

\begin{abstract}
A numerical investigation on spray-wall interaction in a heated duct air-flow is reported in the present study. This study is relevant to the Selective Catalytic Reduction (SCR) application for exhaust gas after-treatment in diesel engines. A spin-type static mixer was used to promote droplet mixing following impingement. The effect of mixer blade orientation on the postimpingement droplets is studied. In addition, the focus is also on the effect of liquid preheating. These aspects have not gained much attention in the past studies. A pulsed type spray injection is employed to inject urea water solution (UWS) upstream of the mixer. All spray simulations are performed using ANSYS Fluent V18.0. Here, the continuous phase is modeled using ReynoldsAveraged Navier Stokes (RANS) equation, while the discrete phase model (DPM) is used to generate the spray injection. For modeling the droplet breakup process and spray-wall interaction, Taylor Analogy Breakup (TAB) and Kuhnke wall film models are enabled, respectively. The spray computations are validated against experiments under atmospheric conditions. It is observed that the mixer orientation strongly influences the spatial droplet distribution postimpingement and mixing. When the preheated spray is injected, a reduction in droplet size occurs downstream of the mixer. It enhances the water vaporization process, which leads to faster ammonia formation in a short span of time. Also, the liquid film thickness is found to decrease with an increase in initial liquid temperature.
\end{abstract}

\section{Keywords}

Mixer, SCR, DPM, Spray-wall interaction, Ammonia concentration

\section{Introduction}

Diesel engines are one of the most widely used engines in automotive applications because of their better performance, high thermal efficiency, and torque capacity. But a major issue is emission of harmful Nitrogen-Oxide $\left(\mathrm{NO}_{x}\right)$ gas [1]. In order to facilitate better exhaust gas after-treatment technique to reduce the $\mathrm{NO}_{x}$ gas emission, the Selective Catalytic Reduction (SCR) system is widely adopted because of its higher $\mathrm{NO}_{x}$ conversion efficiency [2, 3]. This approach is based on the injection of urea water solution (UWS) into the hot exhaust gas in the duct upstream of the catalyst. The water evaporates initially, followed by urea thermolysis and hydrolysis of isocyanic acid. It generates the reducing agent ammonia $\left(\mathrm{NH}_{3}\right)$, which interacts with $\mathrm{NO}_{x}$ resulting in harmless by-products such as nitrogen $\left(\mathrm{N}_{2}\right)$ and water $\left(\mathrm{H}_{2} \mathrm{O}\right)$ [4]. The primary requirements of attaining higher de-NOx efficiency are (i) complete thermal decomposition of urea and (ii) uniform spatial distribution of $\mathrm{NH}_{3}$ before it reaches the catalyst entrance. However, these objectives are challenging to achieve, especially when the exhaust gas temperature is low [5]. Further, wall filming should be avoided as it leads to solid deposition resulting in additional back-pressure in the duct. Therefore, for this purpose, different types of mixer are adopted to enhance the droplet breakup process and attain an optimized mixing length $[6,7]$. When the mixer is used in the duct, a large number of smaller droplets and an increase in ammonia conversion rate can be observed compared to the case, where the mixer is absent [8]. When the spray droplets are impinging the mixer blade wall, a complex fluid dynamic process occurs. Hence, a detailed understanding of spray-wall interaction, and the behaviour of postimpingement droplets, that interacts with the air flow past the mixer, is challenging. It should be noted that the afore-mentioned factors play a vital role in the SCR system performance. 
Even though, a channel with circular cross section is related to the practical scenario, most of the past experimental studies were reported using a rectangular cross-section for the ease of optical accessibility. Further, a detailed analysis of numerical solution, focusing on the evolution of post-impingement droplets inside the channel, is essential as it is often difficult to obtain detailed information using experiments.

In the present study, a channel with a circular cross-section is employed to mimic the exhaust duct. A spin-type mixer is adopted and placed downstream of the injector tip such that injected droplets directly impinge on the mixer blade walls. All the simulation cases are carried out using ANSYS Fluent V18.0. An Eulerian and Lagrangian frame of reference is considered that couples the continuous and discrete phases respectively. Standard k- $\omega$ model is used to simulate the turbulence effects in the continuous phase, while the Discrete Random Walk (DRW) model is used to take into account the effect of turbulence in discrete phase. Here, the focus is on two different aspects. First, to study the influence of orientation of the mixer blades relative to the spray jets. Second, to investigate the preheated liquid injection and wall interaction. Both these aspects are important but have not yet been addressed in detail in the literatures. The numerical results of spray characteristics inside the channel are validated against the experimental results corresponding to atmospheric injection conditions reported by Purkar [9]. The dimensions of the domain in the simulation is adopted from the experimental setup of the above paper.

\section{Numerical Methodology Simulation setup}

Figure 1(a) depicts the schematic of the computational domain used in the numerical simulation. A circular channel with $65 \mathrm{~mm}$ diameter and $650 \mathrm{~mm}$ length is considered. On the channel wall, a four-hole injector (Figure $1(\mathrm{a})$ ) is mounted with $30^{\circ}$ inclination angle (with respect to the air-flow direction) to inject a pulsed spray into the cross air-flow stream (refer to Figure 1(a)). The injector exit contains four holes, and each has $200 \mu \mathrm{m}$ diameter. At location L1 $(60 \mathrm{~mm}$ downstream of the injector), a spin type mixer is mounted as in the experiments by Purkar [9]. The spatial droplet distribution is studied at locations L2 and L3 at $180 \mathrm{~mm}$ and $500 \mathrm{~mm}$ downstream of the injector respectively. Here, the mixer swirl number is $S=0.2$ corresponding to the blade angle, $\alpha=17^{\circ}$. Figure 1 (b) represents the front-view of the mixer for four different orientation, $\theta=0^{\circ}, 15^{\circ}, 30^{\circ}$ and $45^{\circ}$, where $\theta$ is defined in the anti-clockwise direction. Table 1 summarizes the physical conditions of air-flow and liquid injection. In the experimental analysis [9], the results for open spray characteristics, spatial droplet distribution along the cross-sectional plane at location L2 and L3 has been reported. Accordingly, the droplets at the same locations are considered in the present simulations as well.

The parametric variations used in the present simulation work are presented in Table 2. As shown in Figure $1(\mathrm{~b})$, the mixer blade position $\left(\theta=0^{\circ}\right)$ is considered in Case-1, where the mixer blade (blade-1 and blade-4) orientation is precisely horizontal. Compared to Case-1, the orientation of mixer blade in Case-2, Case- 3 , and Case- 4 are rotated $15^{\circ}, 30^{\circ}$, and $45^{\circ}$ respectively (refer to Figure 1(b)). Except this, the other properties remains constant among the first four cases (Case-1 to Case-4) as mentioned in Table 2. The reason for adopting the above conditions is to investigate the hydrodynamic aspects of the droplet behavior post-impingement due to different mixer blade orientation. In Case-5 and Case-6, the air flow at higher ambient temperature of $500 \mathrm{~K}$ is considered, while the liquid injection temperature is higher in the latter (refer to Table 2). These conditions are selected to investigate the preheated liquid injection effect on spray-wall interaction as well as to study the evolution of ammonia and water vapor mass fraction.

\section{Numerical modeling}

In this study, all cases were simulated using a coupled Eulerian-Lagrangian framework in ANSYS Fluent V18.0. The Reynolds-Averaged Navier Stokes (RANS) equation is used to model 


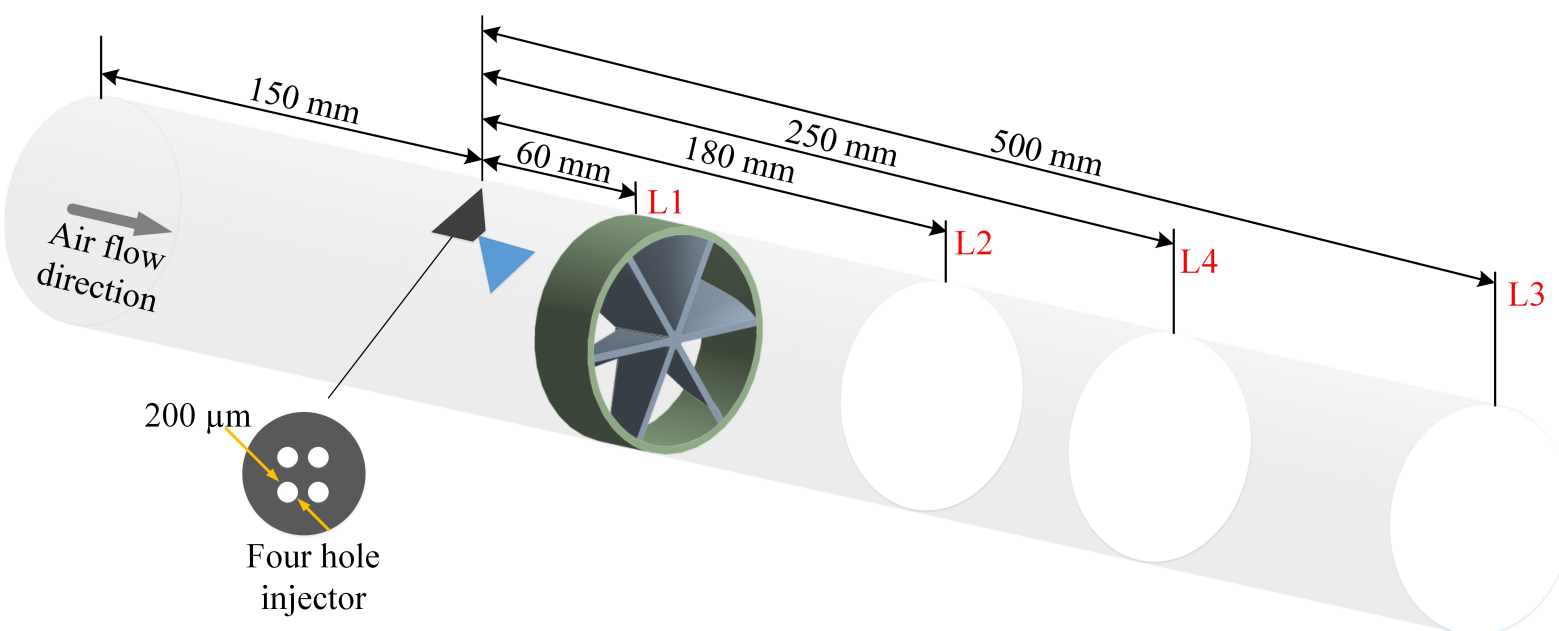

(a)

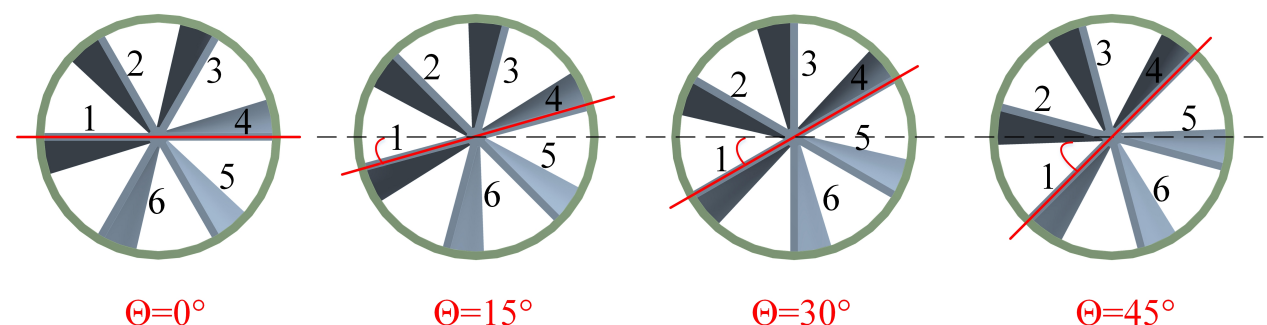

(b)

Figure 1. Schematic of the computational domain (a) Circular channel with spray injection upstream of the mixer and cross-sectional plane locations L2, L3 and L4 (b) Spin type static mixer $\left(S=0.2, \alpha=17^{\circ}\right)$ with four different blade orientation

Table 1. Injection conditions for air and liquid flow

\begin{tabular}{c|lc}
\hline Air flow & Mass flow rate $(\mathrm{kg} / \mathrm{h}), \dot{m}_{\text {air }}$ & 193 \\
\hline \multirow{3}{*}{ Liquid flow } & Injection pressure, $P_{i n j}(\mathrm{bar})$ & 2.8 \\
& Injection duration, $T_{i n j}(\mathrm{~ms})$ & 5 \\
& Mass flow rate, $\dot{m}_{i n j}(\mathrm{mg} / \mathrm{injection})$ & 13
\end{tabular}

the continuous phase, while the discrete phase is modeled using the Discrete Phase Model (DPM).

Continuous phase modeling: To simulate the turbulence effect in continuous phase, the standard k- $\omega$ model is used. The Reynolds-averaged mass conservation equation is given by,

$$
\frac{\partial \rho}{\partial t}+\frac{\partial\left(\rho u_{i}\right)}{\partial x_{i}}=S_{m}
$$

where $S_{m}\left(=\frac{1}{\Delta V} \sum_{n} \dot{m}_{d}\right)$ indicates the volumetric source term (i.e., mass transfer from liquid droplets to the gas phase). Here, $\Delta V$ represents the volume of the cell, while $\dot{m}_{d}$ denotes the droplet evaporation rate. The Reynolds-averaged momentum equation is represented as,

$$
\frac{\partial\left(\rho u_{i}\right)}{\partial t}+\frac{\partial}{\partial x_{j}}\left(\rho u_{i} u_{j}\right)=-\frac{\partial p}{\partial x_{i}}+\frac{\partial}{\partial x_{j}}\left(\mu \frac{\partial u_{i}}{\partial x_{j}}\right)+\frac{\partial}{\partial x_{j}}\left(\overline{\left.-\rho u_{i}^{\prime} u_{j}^{\prime}\right)}+S_{f}\right.
$$

where $\rho$ and $u_{i}$ is the density of the fluid, and ensemble averaged velocity vector respectively. Additionally, $p, \mu$ and $u_{i}^{\prime}$ denotes the fluid pressure, fluid dynamic viscosity, and velocity fluctuation respectively, while $S_{f}\left(=\frac{1}{\Delta V} \sum_{n}\left(\dot{m}_{d} u_{i}-F_{D, u_{i}}-F_{\Delta P_{i}}\right)\right)$ indicates the momentum source 
Table 2. Parametric variations used in the present numerical simulation investigation

\begin{tabular}{c|cccc}
\hline & $\begin{array}{c}\text { Blade orientation in } \\
\text { central part of the mixer with } \\
\text { referring horizontal direction }\left(^{\circ}\right)\end{array}$ & $\begin{array}{c}\text { Temperature of } \\
\text { air flow, } \mathrm{T}_{\text {air }}(\mathrm{K})\end{array}$ & $\begin{array}{c}\text { Temperature of } \\
\text { injecting liquid, } \mathrm{T}_{\text {inj }}(\mathrm{K})\end{array}$ & $\begin{array}{c}\text { Liquid } \\
\text { material }\end{array}$ \\
\hline Case-1 & 0 & 300 & 300 & Water \\
Case-2 & 15 & 300 & 300 & Water \\
Case-3 & 30 & 300 & 300 & Water \\
Case-4 & 45 & 300 & 300 & Water \\
Case-5 & 0 & 500 & 300 & UWS \\
Case-6 & 0 & 500 & 340 & UWS
\end{tabular}

term (i.e. momentum exchange between the spray-droplets and the gas phase). In this term, $F_{D, u_{i}}$ denotes the drag and $F_{\Delta P_{i}}$ represents the pressure gradient forces in the $i^{\text {th }}$ direction respectively. The following equation (3) represents the energy conservation as,

$$
\frac{\partial(\rho E)}{\partial t}+\frac{\partial}{\partial x_{j}}\left(u_{j}(\rho E+P)\right)=\frac{\partial}{\partial x_{j}}\left(K_{e f f} \frac{\partial T_{\infty}}{\partial x_{j}}+u_{i} T_{i j, e f f}\right)+S_{h}
$$

where $E, K_{\text {eff }}$ and $T_{i j, e f f}$ indicates the total energy, effective thermal conductivity and deviatoric stress tensor respectively. The source term $S_{h}\left(=\frac{1}{\Delta V} \sum_{n}\left(\dot{m}_{p} C_{p_{p}}\left(T_{\infty}-T_{p}\right)+\dot{m}_{p} H_{\text {latent }}\right)\right)$ denotes the heat exchange between the gas phase and the droplets. In this term, $T_{\infty}$ and $T_{p}$ are the temperatures of the gas phase and the droplets respectively, while $H_{\text {latent }}$ is the latent heat of the injected liquid.

Discrete phase modeling: In the present work, a plain orifice atomizer model is adopted for injecting the liquid spray. Here, the range of droplet size follows the Rosin-Rammler Diameter (RRD) distribution and can be represented as,

$$
D=\bar{d}\left(-\ln (1-Y)^{\frac{1}{n}}\right)
$$

where $Y$ represents the mass fraction which is small compared to the droplet diameter $(D)$, while $n$ is the exponent value used for the measurement of spread. A large value of $n$ denotes a uniform spray, and the Rosin-Rammler diameter is represented by $\bar{d}$.

The $D^{2}$-law model is enabled to simulate the droplet evaporation in this study. Here, it is important to consider the effect of convective heat transfer from the spray droplet surface to the infinite medium (bulk gas phase). Accordingly, the rate of change of droplet mass, $\dot{m}_{p}$ can be estimated from the following equation,

$$
\dot{m}_{p}=\rho_{p} A_{p} k_{e} \ln \left(1+B_{y}\right)
$$

where $A_{p}$ and $k_{e}$ denotes the droplet surface area and mass transfer coefficient respectively. The term $B_{y}\left(=\frac{Y_{A, S}-Y_{A, \infty}}{1-Y_{A, S}}\right)$ on the right hand side of equation (5) represents the Spalding mass transfer number. Here, $Y_{A, S}$ is the mass fraction of the liquid vapour on the droplet surface and $Y_{A, \infty}$ is the mass fraction of the gas phase.

$$
S h=\frac{K_{e} D_{p}}{D_{v}}=2+0.6 R e^{0.5} S c^{0.33}
$$

where $D_{v}$ is diffusion coefficient of water vapor in the infinite medium, while $D_{p}$ represent the droplet diameter. In the above equation (6), Reynolds and Schmidt numbers are denoted by $R e$ and $S c$, respectively. By using Clausius-Clapeyron equation, the vapour mass fraction was calculated. Note that the vapour pressure is considered as piece-wise linear function of the 
temperature. In order to estimating the vapor-gas mixture properties, the following assumptions were made (i) incomprssible flow (ii) ideal gas and (iii) uniform droplet temperature.

When the UWS is injected inside the channel, it reacts with exhaust gas, decomposes and produces $\mathrm{NH}_{3}$ through two major process (i) thermolysis and (ii) hydrolysis. For modeling the thermal decomposition of UWS, the kinetic rate model is employed. Accordingly, the following Arrhenius kinetic rate expression is used and the constant values presented in the equation 7 were obtained from the literature paper reported by Kim et al [10].

$$
K=A \cdot \exp \left(\frac{-E_{a}}{\bar{R} T}\right)
$$

where $A$ is the pre-exponential factor $\left(S^{-1}\right)$ which is taken as 382 for thermolysis and $2.5 \mathrm{x}$ $10^{5}$ for hydrolysis, while $E_{a}$ is the activation energy $(\mathrm{KJ} / \mathrm{kmol})$ considered as $2.94 \times 10^{7}$ and $6.22 \times 10^{4}$ for thermolysis and hydrolysis process respectively. The process of droplet breakup, collision and collision are modelled using the TAB, stochastic collision and coalescence models respectively. In order to take into account the effect of instantaneous turbulent velocity fluctuations on the spray droplets, Discrete Random Walk (DRW) model is enabled. For spray-wall interaction effect, Kuhnke wall-film is used. More details of spray-wall interaction studies can be found in the article reported by Kuhnke [11]. It should be noted that the steady air-flow is developed across the entire channel before enable the unsteady spray injection.

The domain is discretized using hexahedral type mesh. Further, a grid independence study is conducted with the cell sizes of $1 \times 1 \mathrm{~mm}, 1.5 \times 1.5 \mathrm{~mm}, 2 \times 2 \mathrm{~mm}$ and $2.5 \times 2.5 \mathrm{~mm}$. In the present work, the results of $1.5 \times 1.5 \mathrm{~mm}$ grid cell size is found to be the optimum size for which the results were in close agreement with the experiments. Hence, it is adopted to carry out all the cases in simulation.

\section{Validation}

\section{Open spray characteristics}

Initially, the spray computation results (spray structure, spray tip penetration length, and droplet size) for injection under open atmosphere are validated against the corresponding experimental data to understand the spray behavior. It is noted that the simulation results are in close agreement with the experiment from the comparison figures, which are not reported here. The mean droplet size is measured at $60 \mathrm{~mm}$ downstream of the injector tip in experiment. Accordingly, the results are obtained at the same location in the simulation as well. It was found that the simulation results of $\mathrm{AMD}(\approx 165 \mu \mathrm{m})$ and $\operatorname{SMD}(\approx 203 \mu \mathrm{m})$ agree well with experimental results, where AMD and SMD are reported as $156 \mu \mathrm{m}$ and $214 \mu \mathrm{m}$, respectively.

\section{In-channel spray characteristics}

First, the swirling air-flow past the mixer is validated inside the channel with no spray injection. The velocity modification of airflow past the mixer and swirl flow decay at the downstream location of the mixer is examined. The swirling air-flow direction can be observed as clockwise (refering to the domains front view in Figure 1(a)). Figure 2(a) shows the quantitative comparison of the swirl flow velocity component between simulation and experiment. Here, the simulation results of tangential velocity component are validated against experiment. From this comparison, it was found that the simulation results are in close agreement with experiment in terms of both magnitude and overall radial trend. Next, the spatial droplet distribution at different axial locations (L2 and L3 - refer to Figure 1(a)) are investigated and validated with experimental results. Figure 2(b) shows the spatial droplet distribution along the cross-sectional plane at location L2 and L3 downstream of the mixer. In this figure, two groups of droplets with a large number density are spotted at location L2, one at the top-right side portion and other at the bottom portion of the channel wall. It is mainly caused by the strong influence of swirl flow on the spray droplets after impingement. Considering the droplet distribution at location L3, it is uniformly distributed along the circumference of the channel, while void space 
is observed at the channel centre portion. When the simulation results are compared with experiment as shown in Figure 2(b), the trend followed by the simulation is found to be similar to the experiment and also the reasonable agreement is noticed.

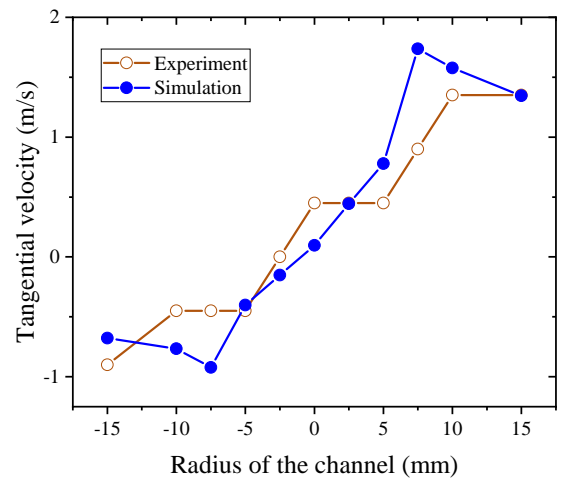

(a)
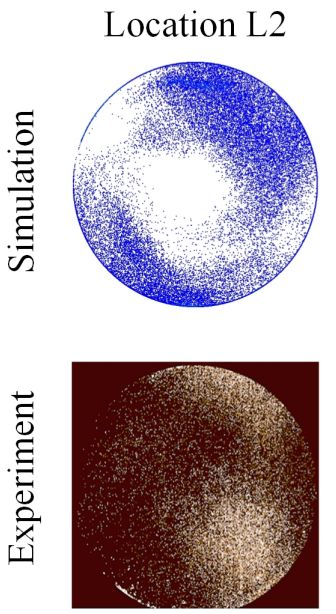

(b)

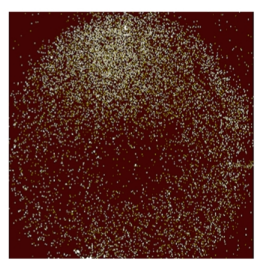

Location L3

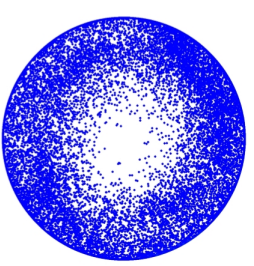

Figure 2. In-channel validation (a) Radial evolution of tangential velocity component of air-flow at the channel outlet (b) Comparison of post-impingement spatial droplet distribution at different axial location in-channel between simulation and experiment

\section{Results and discussion \\ Effect of mixer blade orientation}

This section presents the results pertaining to different orientation of the mixer blades on the post-impingement behavior of the droplets. The time sequence of the spray impingement process followed by droplet mixing for different mixer blade orientation cases are depicted in Figure 3. Note that the colour bar indicate droplet sizes in this figure. In order to clearly visualize the impingement of spray plumes on the mixer blade wall, an isometric view is presented just before impingement (refer to the first column in Figure 3 at $\Delta \mathrm{t}=3 \mathrm{~ms}$ ). It shows that the impingement of spray plumes on both side of the mixer blade wall occurs in Case-2 compared to other cases. In both Case-1 and Case-4, the spray plume is observed to be completely impinges the left side of the mixer blade, while some of the droplets directly hit the channel wall on the bottom-right side of the mixer (refer to $5^{t h} \mathrm{~ms}$ at Figure 3). Unlike the above cases, a higher concentration of larger droplet sizes is spotted on the bottom-right side portion of the mixer in Case-3. As the spray droplets are impinging the blade wall, it is resulted in deposition and splashing. The liquid film on the blade wall is caused by the droplet depostion, while the splashing droplets are tracing the swirl flow path (refer to $7^{\text {th }} \mathrm{ms}$ at Figure 3 ). It is also observed that few droplets are directly carried away by the air-flow without hitting the mixer blade wall. As time progresses (for instance, $\Delta \mathrm{t}=20 \mathrm{~ms}$ ), the spatial droplet distribution is observed to be uniform towards the channel periphery in Case-1 and Case-2, while a smaller droplet concentration is appeared at the left portion of the channel in Case-4 (refer to $20^{\text {th }}$ ms at Figure 3 ). Considering the spatial droplet distribution in Case-3 for $\Delta \mathrm{t}=20 \mathrm{~ms}$, it is observed that a large number of bigger droplet sizes are spotted at the channel centre portion, when compared to the other cases. This is attributed to the impingement of large number of droplets on the channel wall without hitting the mixer blade. Further, the mean droplet size and wall film thickness at mixer downstream locations are examined in Case-1 to Case-4, which are not shown here. When the above results are compared, the droplet size as well as film thickness are noticed to be smaller in Case-1 compared to other cases. It is attributed to the impingement of spray droplets on both side of the mixer blade, which enhances droplet breakup process and produce large number of smaller droplets post-impingement. Therefore, the configuration of mixer blade orientation 
used in Case-1 is preferable.

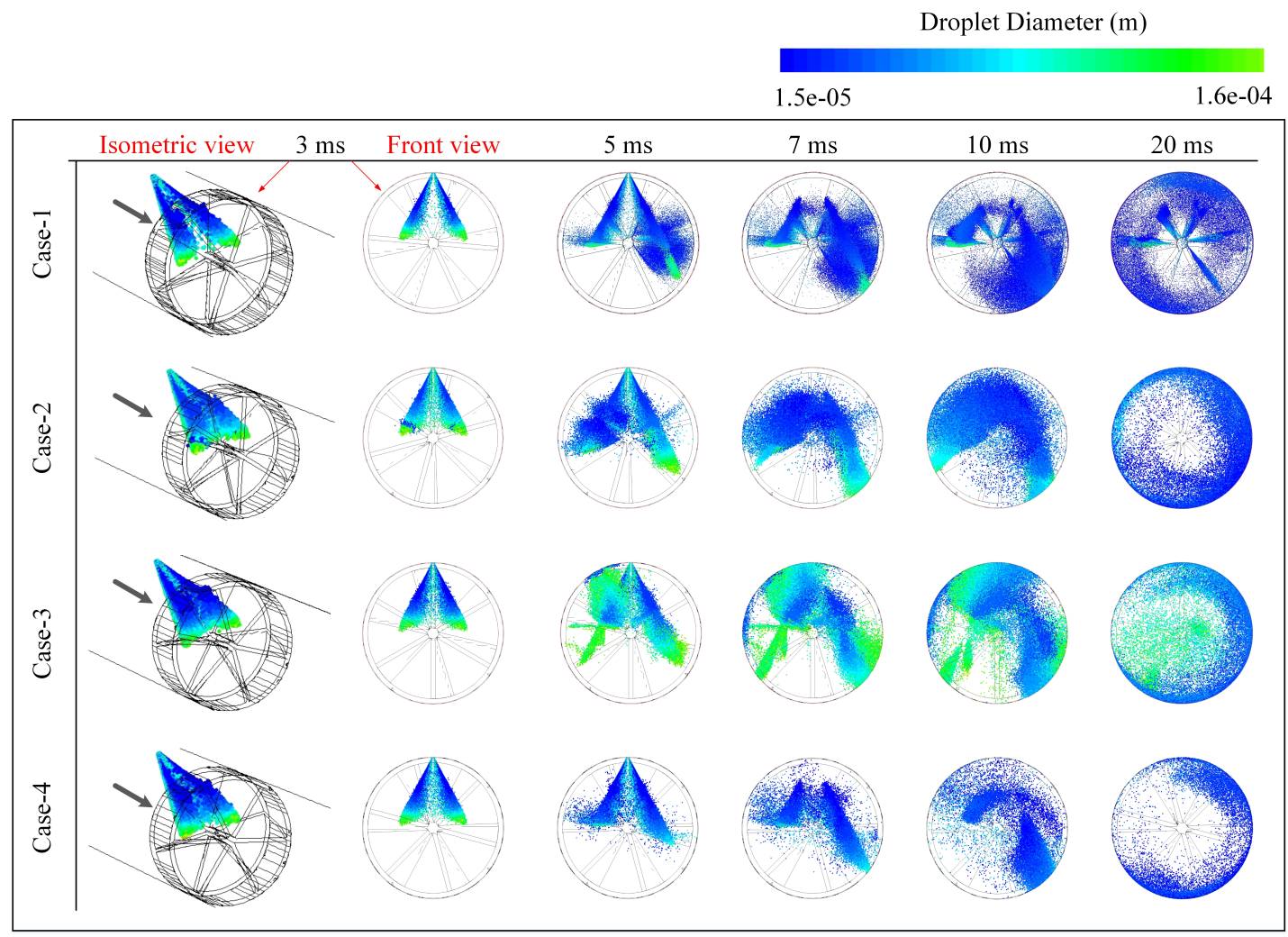

Figure 3. Time evolution of spray impingement in different mixer blade orientation simulation cases $\left(\right.$ Case- $1=0^{\circ}$, Case $-2=15^{\circ}$, Case $-3=30^{\circ}$, Case $-4=45^{\circ}$ )

\section{Effect of preheated liquid injection}

In this section, the influence of liquid injection at an elevated temperature condition on postimpingement droplet size distribution, $\mathrm{H}_{2} \mathrm{O}$ vapor and $\mathrm{NH}_{3}$ concentration, and wall-filming are investigated. First, the spatial droplet distribution is studied at the injector downstream locations (L2 and L3) as depicted in Figure 4(a). It shows that the droplet number density is smaller for both locations in Case-6 compared to Case-5. This is attributed to enhanced water vapor concentration, which causes the reduction in droplet size as a result of preheating the liquid. It is also evident in the SMD comparison plot between Case- 5 and Case- 6 as shown in Figure 4(b). The droplet size is found to decrease with an increase in temperature of the injected liquid. Second, the $\mathrm{H}_{2} \mathrm{O}$ vapor fraction and $\mathrm{NH}_{3}$ concentration are investigated at the injector downstream locations as depicted in Figure 5. In Figure 5(a), the $\mathrm{H}_{2} \mathrm{O}$ vapor fraction is observed to be higher in Case-6, when compared to Case-5. It mainly arises due to enhanced $\mathrm{H}_{2} \mathrm{O}$ vaporization process, which leads to faster formation of $\mathrm{NH}_{3}$ in a short span of time. This occurs because of faster urea thermal decomposition after water gets vaporized. It is also confirmed in Figure 5(b), where $\mathrm{NH}_{3}$ concentration is found to be higher in Case-6 compared to Case-5. Also, the uniformity of $\mathrm{NH}_{3}$ concentration along the cross-sectional plane at mixer downstream locations is examined, which is not reported here. In Case-6, due to the liquid preheating, the $\mathrm{NH}_{3}$ concentration is started to appear closer to the injector tip in a short span of time after injection starts, compared to Case-5. It is also noticed that the spatial uniformity of $\mathrm{NH}_{3}$ concentration is better in Case-6, when compared with Case-5. Figure 6 depicts the comparison of time evolution of averaged liquid film thickness over the entire channel between Case-5 and Case-6. In this figure, an increasing trend in film thickness is observed for the initial time instants (i.e., 5 to $11 \mathrm{~ms}$ ). But once the impingement is over at $\Delta \mathrm{t} \approx 12 \mathrm{~ms}$, a slight reduction in the thickness is observed due to the wall filming is influenced by air-flow. This is also attributed to the heat transfer that takes place between channel wall and liquid deposition. 
Beyond $\Delta \mathrm{t} \approx 14 \mathrm{~ms}$, a reduction in film thickenss is found to be minimal. When this results are compared between Case-5 and Case- 6 as shown in Figure 6, the film thickness is noted to be smaller in Case-6. It mainly occurs due to the enhanced liquid-film evaporation rate. Therefore, this investigation reveals that an increase in liquid injection temperature leads to (i) reduction in droplet size and film thickness and (ii) enhance $\mathrm{H}_{2} \mathrm{O}$ vapor fraction and $\mathrm{NH}_{3}$ concentration.

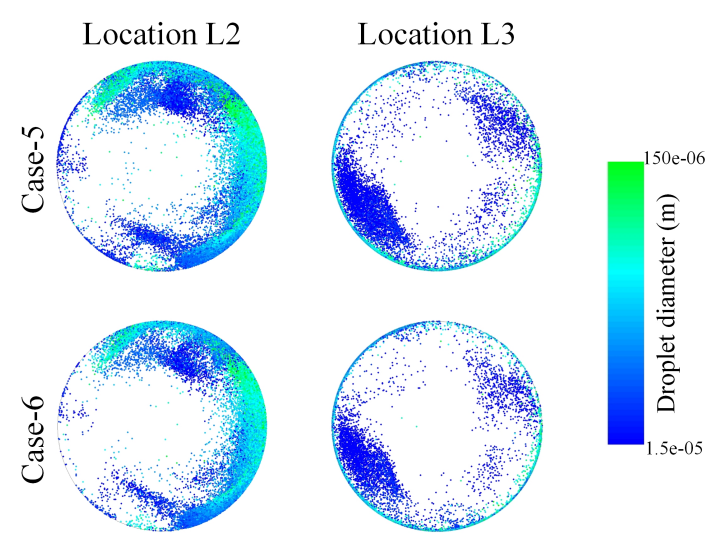

(a)

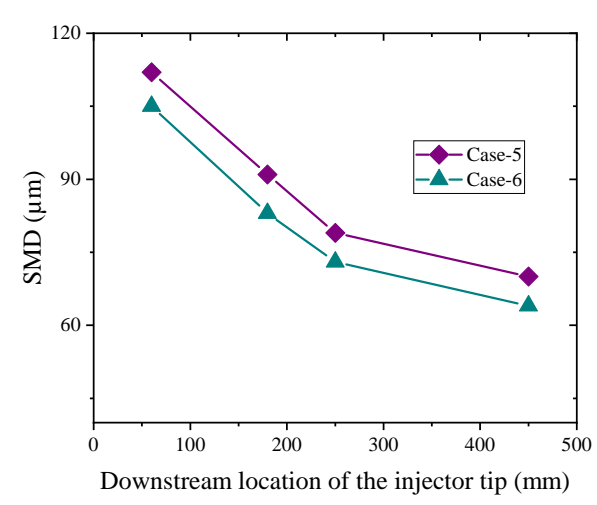

(b)

Figure 4. Comparison of droplet size distribution at injector downstream locations between Case- 5 and Case-6 (a) post-impingement spatial droplet distribution at location L2 and L3 (b) Sauter Mean Diameter at pre and post-impingement locations

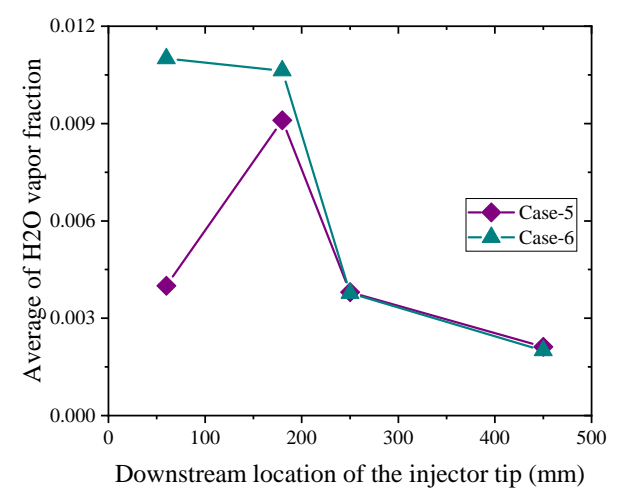

(a)

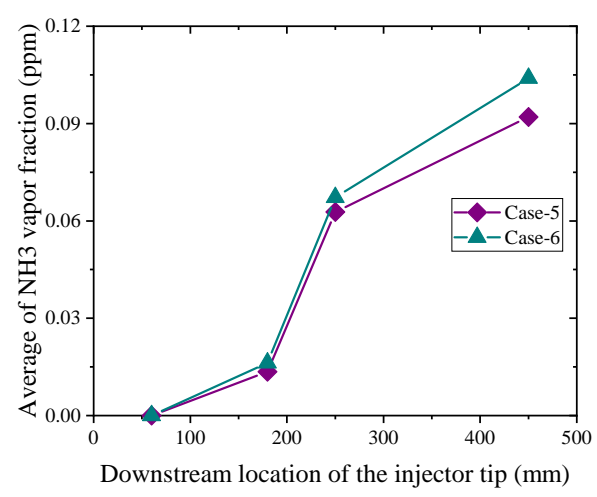

(b)

Figure 5. Comparison of averaged (a) $\mathrm{H}_{2} \mathrm{O}$ vapor fraction and (b) $\mathrm{NH}_{3}$ concentration at injector downstream locations between Case- 5 and Case- 6

\section{Conclusions}

A numerical investigation of spray-wall interaction and mixing was carried out for a pulsed spray injection into heated air flow in a duct, which is relavent to SCR applications. The present study is investigated based on two different aspects. First, the mixer blade orientation effect relative to the spray plume jets is studied under atmospheric conditions. It reveals that the spatial droplet distribution after impingement and mixing are strongly influenced by the swirling air-flow generated by the mixer. Also, it was found that the orientation of the mixer blade at different position causes the signifcant difference in the spray droplets behavior after impingement. Second, the influence of preheated liquid injection on the droplet size trend after impingement, $\mathrm{H}_{2} \mathrm{O}$ vapor fraction and $\mathrm{NH}_{3}$ concentration, and liquid film thickness is investigated. It shows that with an increase in temperature of the injected liquid, the droplet size and film thickness are found to be reduced, while $\mathrm{H}_{2} \mathrm{O}$ vapor fraction, and concentration of $\mathrm{NH}_{3}$ are increased. It is mainly 


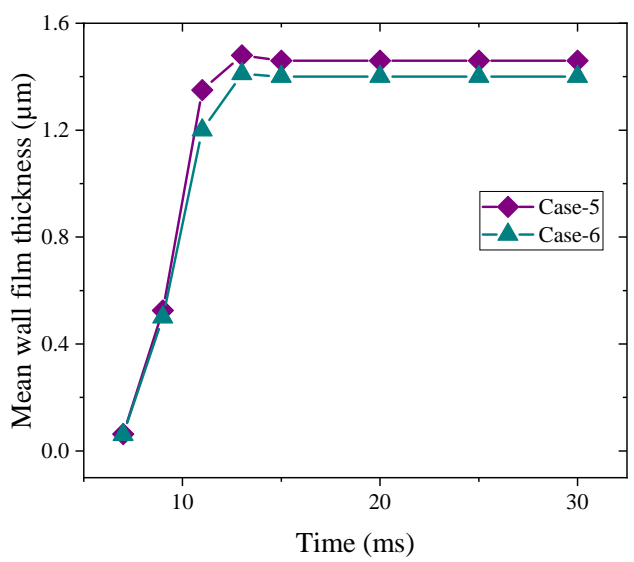

Figure 6. Comparison of time evolution of mean wall film thickness between Case- 5 and Case- 6

caused by enhanced water vaporization process. Also, it is noticed that the formation of $\mathrm{NH}_{3}$ is achieved in a short span of time and shorter distance due to preheating the liquid. Further, the present work will be extended to study the effect of a large increase in initial temperature of the liquid injection on spray-wall interaction, $\mathrm{NH}_{3}$ concentration and uniformity index.

\section{References}

[1] Heywood, J. B., 2018," Internal combustion engine fundamentals", McGraw-Hill Education.

[2] Gieshoff, J., Schäfer-Sindlinger, A., Spurk, P. C., Van Den Tillaart, J. A. A., and Garr, G., 2000, "Improved SCR systems for heavy duty applications," No. 0148-7191, SAE Technical Paper.

[3] Fang, H. L., and DaCosta, H. F. M., 2003, "Urea thermolysis and NOx reduction with and without SCR catalysts," Applied Catalysis B: Environmental, 46(1), pp. 17-34.

[4] Koebel, M., Elsener, M., and Marti, T., 1996, "NOx-reduction in diesel exhaust gas with urea and selective catalytic reduction," Combustion science and technology, 121(1-6), pp. 85-102.

[5] Varna, A., Spiteri, A. C., Wright, Y. M., Eggenschwiler, P. D., and Boulouchos, K., 2015, "Experimental and numerical assessment of impingement and mixing of urea-water sprays for nitric oxide reduction in diesel exhaust," Applied Energy, 157, pp. 824-837.

[6] Oh, J., Kim, K., and Lee, K., 2012, "Effects of various mixer types on the spatial distribution of a De-NOx reductant," Fuel, 94, pp. 93-101.

[7] Oh, J., and Lee, K., 2014, "Spray characteristics of a urea solution injector and optimal mixer location to improve droplet uniformity and NOx conversion efficiency for selective catalytic reduction," Fuel, 119, pp. 90-97.

[8] Tan, L., Feng, P., Yang, S., Guo, Y., Liu, S., and Li, Z., 2018, "CFD studies on effects of SCR mixers on the performance of urea conversion and mixing of the reducing agent," Chemical Engineering and Processing-Process Intensification, 123, pp. 82-88.

[9] Purkar, K. A., 2019, "Optical characterization of spray impingement and mixing in a channel for SCR application," Thesis, Indian Institute of Technology Madras.

[10] Kim, J. Y., Ryu, S. H., and Ha, J. S., "Numerical prediction on the characteristics of sprayinduced mixing and thermal decomposition of urea solution in SCR system," pp. 165-170.

[11] Kuhnke, D., 2004, Spray/wall interaction modelling by dimensionless data analysis, Shaker. 